$\angle$ Research Square

\section{DNA Barcoding Provides Insights Into Fish Diversity and Molecular Taxonomy of the Amundsen Sea}

Shuai Cao

Shanghai Ocean University

Yuan Li

Third Institute of Oceanography Ministry of Natural Resources

\section{Xing Miao}

Third Institute of Oceanography Ministry of Natural Resources

\section{Ran Zhang}

Third Institute of Oceanography Ministry of Natural Resources

Longshan Lin ( $\square$ linlongshan@tio.org.cn)

Third Institute of Oceanography Ministry of Natural Resources https://orcid.org/0000-0002-3646-0426

Hai Li

Third Institute of Oceanography Ministry of Natural Resources

\section{Research Article}

Keywords: Antarctic fish, Amundsen Sea, DNA barcode, COI gene, fish diversity, taxonomy

Posted Date: January 21st, 2022

DOI: https://doi.org/10.21203/rs.3.rs-1244705/v1

License: (c) This work is licensed under a Creative Commons Attribution 4.0 International License. Read Full License 


\section{Abstract}

The Southern Ocean is experiencing complex climate change, and the Amundsen Sea is one of the regions that responds most rapidly to climate change. Due to their role in ecosystems, environmental sensitivity and high endemism, Antarctic demersal fish are a favourable group that can act as an indicator to indicate the response of Antarctic organisms to climate change. However, our knowledge of Antarctic fish fauna is insufficient, with knowledge gaps even in their taxonomy. This situation is greatly influenced by the limitations of traditional taxonomy and thus calls for alternative solutions such as DNA barcoding. In this study, DNA barcoding analyses of 69 fish samples obtained from the Amundsen Sea were conducted using the mitochondrial COI gene. Based on molecular species delimitation results, 13 fish species belonged to two orders, six families, and 12 genera. Both the maximum likelihood and Bayesian inference methods showed that the phylogenetic relationships of Bathydraconidae were paraphyletic, which was consistent with previous phylogenetic research. Our research showed that the COI gene, as a DNA barcode, not only is suitable for the identification of Antarctic fish species but also reflects some phylogenetic characteristics that might provide important evidence and support for studies of Antarctic fish phylogenetic relationships. In summary, our study provides an important reference for fish diversity and taxonomy in the Amundsen Sea, which may further enhance our understanding of the biodiversity, taxonomy and biogeography of fish in this area.

\section{Introduction}

The Southern Ocean occupies almost $10 \%$ of the ocean area on Earth (Joyner 1998). It is the only ocean that surrounds Earth and is not divided by continents. This gives it a unique ocean current system. The Antarctic Circumpolar Current (ACC) travels around Antarctica in a clockwise direction, driven by sustained westerly winds (Allison et al. 2010). It prevents warm water from flowing from lower latitudes to higher latitudes, making the Southern Ocean one of the coldest oceans on Earth (Tynan 1998). Organisms in the Southern Ocean have adapted to the polar climate after millions of years of evolution (Clarke \& Johnston 1996). However, complex climate change occurs in Antarctica, especially in West Antarctica, such as the Amundsen Sea (Jun et al. 2020). Changes in the marine environment, especially temperature (McGlone et al. 2010), salinity (Haumann et al. 2016), and dissolved oxygen(Keller et al. 2016), may have important effects on the marine ecosystem and biological community structure (La et al. 2019). As one of the most widely distributed and richest species groups in the ocean, fish are a key component of the marine ecosystem that maintains their balance. They not only serve a basic ecological function but also play an important role in indicating the operating status of the ecosystem (Hunt Jr et al. 2002; Vander Zanden et al. 2011). Modern Antarctic fish fauna, whether in terms of biodiversity, abundance, or biomass, are mainly dominated by Notothenioidei, including Artedidraconidae, Bathydraconidae, Channichthyidae, Harpagiferidae, and Nototheniidae (Mintenbeck et al. 2012). These fish live in cold, oxygen-rich, and stable ocean environments and are highly endemic (Mintenbeck \& Torres 2017). These characteristics, along with the roles the fish play in the ecosystem, make Antarctic fishes a favourable group that can act as an indicator of environmental change in the Southern Ocean.

Compared to the vast ocean area of the Southern Ocean, there are only approximately 370 species of fish described which account for $\sim 2 \%$ of all fish species worldwide, nevertheless, this number is underestimated (Eastman 2000). Ice cover, lack of deep-sea samples, low sampling frequency and insufficient traditional taxonomy may also be the reasons for the underestimation of the number (Alt et al. 2021). Unfortunately, the situation of the fish fauna of the Amundsen Sea is even worse, because the Amundsen Sea is located in a remote location relative to scientific research stations and routes (Griffiths et al. 2011). There were only limited observation records and an underwater observation survey report (Eastman et al. 2012), while studies based on molecular taxonomy have not yet been reported. Currently, the Amundsen Sea is among the places where the sea temperature in the Southern Ocean rises most obviously (Kim et al. 2021). The rapid rise in sea temperature has led to a decrease in sea ice cover and a sustained decline in the ice shelf (Haumann et al. 2016). Meanwhile, the benthic ecosystem in Antarctica is vulnerable (Pineda-Metz et al. 2020), glacier retreat (Sahade et al. 2015) and associated iceberg scouring (Gutt \& Piepenburg 2003; Barnes \& Souster 2011) have a huge impact on benthic communities, including Antarctic fish, which mostly belong to demersal fish (Mintenbeck et al. 2012). Moreover, the decline in salinity and dissolved oxygen (Yager et al. 2012; RandallGoodwin et al. 2015) also brings challenges to fish survival that cannot be ignored. As one of the important indicator groups of climate change, the lack of information on the composition of fish communities in the Amundsen Sea will seriously affect the evaluation of the structure and function of its marine ecosystem. Therefore, a fish diversity baseline inventory is urgently needed, and clarifying the characteristics of Amundsen Sea fish diversity patterns can help us better understand the impacts of climate change on Amundsen Sea marine ecosystems.

Traditional fish classification is based on morphological identification, which is time-consuming and depends on the experience of the taxonomist (Steinke et al. 2009). However, the morphologies of sibling species are similar, which can easily lead to misidentification. In particular, the amazing diversity of sizes, colours, and shapes in different life stages of fish is a challenge to taxonomists (Zhang \& Hanner 2012). At the same time, the taxonomic division of some fish in the Southern Ocean is controversial (De Broyer et al. 2014). All these problems require new solutions. The emergence of species identification methods based on molecular biology has given taxonomists more choices and has the potential to become a universal method. This method is expected to become one of the most convincing types of classification evidence (Hebert et al. 2003a). DNA barcoding is increasingly being advocated for in the identification of species. DNA barcoding based on the cytochrome c oxidase subunit I (COI) mitochondrial gene was applied to the identification of species (Hebert et al. 2003b). A COI fragment of $650 \mathrm{bp}$ has enough sequence diversity to reflect significant specieslevel differences and has demonstrated high efficiency and accuracy in species identification on a global scale, such as in Japanese marine fish (Zhang \& Hanner 2011), Indian marine fish (Lakra et al. 2011), Cuban freshwater fish (Lara et al. 2010), Indo-Pacific coral reef fish (Hubert et al. 2012), and even birds (Hebert et al. 2004), mammals (Francis et al. 2010), and bivalves (Mikkelsen et al. 2007), among others. In this paper, the COI-based molecular identification method is applied to Antarctic fish of the Amundsen Sea. Our research aims to provide fundamental taxonomic information for fish species of the Amundsen Sea, and thus provide a solid scientific basis for the ecological assessment and biological conservation of the Southern Ocean. 


\section{Materials And Methods \\ 2.1 Specimen collections}

All specimens were collected at Xuelong icebreaker research vessels during the 36th Chinese National Antarctic Research Expedition (CHINARE) in 2020. Specimens were caught by a bottom trawling net ( $2.2 \mathrm{~m}$ wide, $0.65 \mathrm{~m}$ high, and $6.5 \mathrm{~m}$ long, $20 \mathrm{~mm}$ mesh diameter). Every net was employed for approximately 10 15 minutes at speeds of 2 3 kn. All samples were collected from 4 stations (Fig. 1) in the Amundsen Sea. All caught fish were sorted at $-20^{\circ} \mathrm{C}$ and provisionally identified. Muscle samples were stored in $95 \%$ ethanol for DNA extraction. Finally, all fish were fixed in $10 \%$ formaldehyde and stored as voucher samples at the Third Institute of Oceanography, Ministry of Natural Resources.

\subsection{DNA preparation, PCR and sequencing}

DNA extraction was carried out with muscle tissue by using a DNeasy Blood and Tissue Kit [Qiagen, Hilden, Germany]. Some steps followed those of Hellberg et al (2014). Microtubes of $1.5 \mathrm{~mL}$ [Axygen, New York, American] and ethanol (99.7\%) [XILONG SCIENTIFIC, Guangdong, China] were prepared in advance. Muscle samples (approximately $30 \mathrm{mg}$ ) were weighed into $1.5 \mathrm{~mL}$ microtubes, and then the steps in the manufacturer's instructions were followed. Finally, DNA was stored at $-20^{\circ} \mathrm{C}$ until PCR amplification. The primers in this study were designed by Ward (2005) and were used for COI amplification.

All PCRs had a total volume of $25 \mu \mathrm{L}$ and included $17.25 \mu \mathrm{L}$ of ultrapure water, $2 \mu \mathrm{L}$ of dNTPs ( $2.5 \mathrm{mM}), 2.5 \mu \mathrm{L}$ of $10 \times$ PCR buffer (including Mg ${ }^{2+}$ ) (20 $\mathrm{mM}), 1 \mu \mathrm{L}$ of each primer, $0.25 \mu \mathrm{L}$ of Taq polymerase [TaKaRa, Kusatsu, Japan] $(5 \mathrm{U} / \mu \mathrm{L})$, and $1 \mu \mathrm{L}$ of DNA template. Amplifications were performed using a SensoQuest LabCycler [SensoQuest, Germany] gradient thermal cycler. PCR cycling consisted of an initial step of 4 min at $95{ }^{\circ} \mathrm{C}$ and 35 cycles of $30 \mathrm{~s}$ at $94{ }^{\circ} \mathrm{C}, 30 \mathrm{~s}$ at $50{ }^{\circ} \mathrm{C}$, and $30 \mathrm{~s}$ at $72{ }^{\circ} \mathrm{C}$, followed by a final extension at $72{ }^{\circ} \mathrm{C}$ for $10 \mathrm{~min}$. PCR products were loaded onto $1 \%$ agarose gels and selected for sequencing, and all the PCR products were purified and sequenced by Personal Biotechnology Co., Ltd.

\subsection{DNA identification and phylogenetic analysis}

All COI sequences were edited using DNASTAR Lasergene SeqMan Pro 7.1 and aligned manually using Sequencher 4.1 (Gene Codes 2000 ). All the COI sequences (> $650 \mathrm{bp}$ ) were BLAST searched against the NCBI database and matches with more than $98 \%$ similarity were considered the same species(Murphy et al. 2016). To facilitate the calculation of the genetic distance, two additional data points from the NCBI database were added for each species with fewer than three fish. The newly isolated nucleotide sequences were deposited in GenBank under Accession Numbers (Table 1). 
Table 1

Information of samples and species identification

\begin{tabular}{|c|c|c|c|c|c|c|c|c|c|}
\hline $\begin{array}{l}\text { Sample } \\
\text { No. }\end{array}$ & $\begin{array}{l}\text { Sample } \\
\text { site }\end{array}$ & $\begin{array}{l}\text { Longitude } \\
\text { ( } / \mathrm{W})\end{array}$ & $\begin{array}{l}\text { Latitude } \\
(\% / \mathrm{S})\end{array}$ & $\begin{array}{l}\text { Sample } \\
\text { Depth(m) }\end{array}$ & Molecular identification & $\begin{array}{l}\text { Morphological } \\
\text { identification }\end{array}$ & $\begin{array}{l}\text { Genbank } \\
\text { voucher No. }\end{array}$ & $\begin{array}{l}\text { Similarity } \\
\text { (\%) }\end{array}$ & $\begin{array}{l}\text { Genbank } \\
\text { No. }\end{array}$ \\
\hline AN1 & A11-1 & 113.35 & 73.52 & 627 & Dacodraco hunteri & $\begin{array}{l}\text { Dacodraco } \\
\text { hunteri }\end{array}$ & HQ712963.1 & 99.85 & OK493632 \\
\hline AN2 & A11-4 & 117.32 & 72.25 & 523 & Lycenchelys sp. & $\begin{array}{l}\text { Ophthalmolycus } \\
\text { amberensis }\end{array}$ & EU326372.1 & 99.35 & OK493633 \\
\hline AN3 & A11-4 & 117.32 & 72.25 & 523 & Trematomus scotti & $\begin{array}{l}\text { Trematomus } \\
\text { scotti }\end{array}$ & KX676179.1 & 99.85 & OK493645 \\
\hline AN4 & A11-4 & 117.32 & 72.25 & 523 & Trematomus scotti & $\begin{array}{l}\text { Trematomus } \\
\text { scotti }\end{array}$ & HQ713279.1 & 99.69 & OK493646 \\
\hline AN5 & A11-4 & 117.32 & 72.25 & 523 & Trematomus scotti & $\begin{array}{l}\text { Trematomus } \\
\text { scotti }\end{array}$ & KX676176.1 & 99.54 & OK493647 \\
\hline AN6 & A11-4 & 117.32 & 72.25 & 523 & Trematomus scotti & $\begin{array}{l}\text { Trematomus } \\
\text { scotti }\end{array}$ & KX676176.1 & 99.69 & OK493648 \\
\hline AN7 & A11-4 & 117.32 & 72.25 & 523 & Trematomus scotti & $\begin{array}{l}\text { Trematomus } \\
\text { scotti }\end{array}$ & EU326433.1 & 100.00 & OK493649 \\
\hline AN8 & A11-4 & 117.32 & 72.25 & 523 & Trematomus scotti & $\begin{array}{l}\text { Trematomus } \\
\text { scotti }\end{array}$ & KX676176.1 & 99.85 & OK493650 \\
\hline AN9 & A11-4 & 117.32 & 72.25 & 523 & Trematomus scotti & $\begin{array}{l}\text { Trematomus } \\
\text { scotti }\end{array}$ & KX676176.1 & 100.00 & OK493651 \\
\hline AN10 & A11-4 & 117.32 & 72.25 & 523 & Trematomus scotti & $\begin{array}{l}\text { Trematomus } \\
\text { scotti }\end{array}$ & KX676176.1 & 100.00 & OK493652 \\
\hline AN11 & A11-4 & 117.32 & 72.25 & 523 & Trematomus scotti & $\begin{array}{l}\text { Trematomus } \\
\text { scotti }\end{array}$ & KX676181.1 & 99.85 & OK493653 \\
\hline AN12 & A11-4 & 117.32 & 72.25 & 523 & Trematomus scotti & $\begin{array}{l}\text { Trematomus } \\
\text { scotti }\end{array}$ & KX676176.1 & 100.00 & OK493654 \\
\hline AN13 & A11-4 & 117.32 & 72.25 & 523 & Trematomus scotti & $\begin{array}{l}\text { Trematomus } \\
\text { scotti }\end{array}$ & KX676179.1 & 99.54 & OK493655 \\
\hline AN14 & A11-1 & 113.35 & 73.52 & 627 & $\begin{array}{l}\text { Vomeridens } \\
\text { infuscipinnis }\end{array}$ & $\begin{array}{l}\text { Vomeridens } \\
\text { infuscipinnis }\end{array}$ & HQ713358.1 & 100.00 & OK493677 \\
\hline AN15 & A11-3 & 113.58 & 73.41 & 423 & Trematomus scotti & $\begin{array}{l}\text { Trematomus } \\
\text { scotti }\end{array}$ & KX676176.1 & 100.00 & OK493681 \\
\hline AN16 & A11-3 & 113.58 & 73.41 & 423 & Trematomus scotti & $\begin{array}{l}\text { Trematomus } \\
\text { scotti }\end{array}$ & HQ713279.1 & 99.85 & OK493682 \\
\hline AN17 & A11-3 & 113.58 & 73.41 & 423 & Trematomus scotti & $\begin{array}{l}\text { Trematomus } \\
\text { scotti }\end{array}$ & KX676176.1 & 99.85 & OK493683 \\
\hline AN18 & A11-3 & 113.58 & 73.41 & 423 & Trematomus scotti & $\begin{array}{l}\text { Trematomus } \\
\text { scotti }\end{array}$ & KX676176.1 & 99.85 & OK493684 \\
\hline AN19 & A11-3 & 113.58 & 73.41 & 423 & Trematomus scotti & $\begin{array}{l}\text { Trematomus } \\
\text { scotti }\end{array}$ & KX676176.1 & 100.00 & OK493685 \\
\hline AN20 & A11-3 & 113.58 & 73.41 & 423 & Trematomus scotti & $\begin{array}{l}\text { Trematomus } \\
\text { scotti }\end{array}$ & KX676176.1 & 99.85 & OK493686 \\
\hline AN21 & A11-3 & 113.58 & 73.41 & 423 & Trematomus scotti & $\begin{array}{l}\text { Trematomus } \\
\text { scotti }\end{array}$ & KX676177.1 & 100.00 & OK493687 \\
\hline AN22 & A11-3 & 113.58 & 73.41 & 423 & Trematomus scotti & $\begin{array}{l}\text { Trematomus } \\
\text { scotti }\end{array}$ & KX676177.1 & 99.85 & OK493688 \\
\hline AN23 & A11-3 & 113.58 & 73.41 & 423 & Trematomus scotti & $\begin{array}{l}\text { Trematomus } \\
\text { scotti }\end{array}$ & KX676177.1 & 99.85 & OK493689 \\
\hline AN24 & A11-3 & 113.58 & 73.41 & 423 & Trematomus scotti & $\begin{array}{l}\text { Trematomus } \\
\text { scotti }\end{array}$ & KX676181.1 & 99.85 & OK493690 \\
\hline AN25 & A11-3 & 113.58 & 73.41 & 423 & Trematomus scotti & $\begin{array}{l}\text { Trematomus } \\
\text { scotti }\end{array}$ & KX676176.1 & 99.39 & OK493691 \\
\hline
\end{tabular}

Note: Morphological names in bold are misidentified samples using morphological taxonomy. 


\begin{tabular}{|c|c|c|c|c|c|c|c|c|c|}
\hline $\begin{array}{l}\text { Sample } \\
\text { No. }\end{array}$ & $\begin{array}{l}\text { Sample } \\
\text { site }\end{array}$ & $\begin{array}{l}\text { Longitude } \\
\left({ }^{\circ} / \mathrm{W}\right)\end{array}$ & $\begin{array}{l}\text { Latitude } \\
(\% / S)\end{array}$ & $\begin{array}{l}\text { Sample } \\
\text { Depth(m) }\end{array}$ & Molecular identification & $\begin{array}{l}\text { Morphological } \\
\text { identification }\end{array}$ & $\begin{array}{l}\text { Genbank } \\
\text { voucher No. }\end{array}$ & $\begin{array}{l}\text { Similarity } \\
\text { (\%) }\end{array}$ & $\begin{array}{l}\text { Genbank } \\
\text { No. }\end{array}$ \\
\hline AN26 & A11-3 & 113.58 & 73.41 & 423 & Trematomus scotti & $\begin{array}{l}\text { Trematomus } \\
\text { scotti }\end{array}$ & EU326433.1 & 99.85 & OK493692 \\
\hline AN27 & A11-3 & 113.58 & 73.41 & 423 & Trematomus scotti & $\begin{array}{l}\text { Trematomus } \\
\text { scotti }\end{array}$ & KX676171.1 & 99.69 & OK493693 \\
\hline AN28 & A11-3 & 113.58 & 73.41 & 423 & Trematomus scotti & $\begin{array}{l}\text { Trematomus } \\
\text { scotti }\end{array}$ & HQ713279.1 & 99.54 & OK493694 \\
\hline AN29 & A11-3 & 113.58 & 73.41 & 423 & Trematomus scotti & $\begin{array}{l}\text { Trematomus } \\
\text { scotti }\end{array}$ & EU326433.1 & 100.00 & OK493695 \\
\hline AN30 & A11-3 & 113.58 & 73.41 & 423 & Trematomus scotti & $\begin{array}{l}\text { Trematomus } \\
\text { scotti }\end{array}$ & KX676176.1 & 99.85 & OK493696 \\
\hline AN31 & A11-3 & 113.58 & 73.41 & 423 & Trematomus scotti & $\begin{array}{l}\text { Trematomus } \\
\text { scotti }\end{array}$ & KX676175.1 & 100.00 & OK493697 \\
\hline AN32 & A11-3 & 113.58 & 73.41 & 423 & Trematomus scotti & $\begin{array}{l}\text { Trematomus } \\
\text { scotti }\end{array}$ & KX676176.1 & 100.00 & OK493698 \\
\hline AN33 & A11-3 & 113.58 & 73.41 & 423 & Trematomus scotti & $\begin{array}{l}\text { Trematomus } \\
\text { scotti }\end{array}$ & KX676176.1 & 100.00 & OK493699 \\
\hline AN34 & A11-3 & 113.58 & 73.41 & 423 & Trematomus scotti & $\begin{array}{l}\text { Trematomus } \\
\text { scotti }\end{array}$ & HQ713279.1 & 99.54 & OK493700 \\
\hline AN35 & A11-3 & 113.58 & 73.41 & 423 & Trematomus scotti & $\begin{array}{l}\text { Trematomus } \\
\text { scotti }\end{array}$ & KX676176.1 & 99.85 & OK493701 \\
\hline AN36 & A11-3 & 113.58 & 73.41 & 423 & Trematomus scotti & $\begin{array}{l}\text { Trematomus } \\
\text { scotti }\end{array}$ & KX676177.1 & 100.00 & OK493702 \\
\hline AN37 & A11-3 & 113.58 & 73.41 & 423 & Trematomus scotti & $\begin{array}{l}\text { Trematomus } \\
\text { scotti }\end{array}$ & KX676176.1 & 100.00 & OK493703 \\
\hline AN38 & A11-1 & 113.35 & 73.52 & 627 & Trematomus scotti & $\begin{array}{l}\text { Trematomus } \\
\text { scotti }\end{array}$ & KX676171.1 & 99.69 & OK493704 \\
\hline AN39 & A11-1 & 113.35 & 73.52 & 627 & Trematomus scotti & $\begin{array}{l}\text { Trematomus } \\
\text { scotti }\end{array}$ & KX676177.1 & 100.00 & OK493705 \\
\hline AN40 & A11-1 & 113.35 & 73.52 & 627 & Trematomus scotti & $\begin{array}{l}\text { Trematomus } \\
\text { scotti }\end{array}$ & KX676176.1 & 99.69 & OK493706 \\
\hline AN41 & A11-1 & 113.35 & 73.52 & 627 & Trematomus scotti & $\begin{array}{l}\text { Trematomus } \\
\text { scotti }\end{array}$ & KX676173.1 & 99.85 & OK493707 \\
\hline AN42 & A11-1 & 113.35 & 73.52 & 627 & Trematomus scotti & $\begin{array}{l}\text { Trematomus } \\
\text { scotti }\end{array}$ & KX676173.1 & 100.00 & OK493708 \\
\hline AN43 & A4-3 & 112.99 & 72.91 & 438 & Chaenodraco wilsoni & $\begin{array}{l}\text { Chaenodraco } \\
\text { wilsoni }\end{array}$ & NC_039158.1 & 99.09 & OK493709 \\
\hline AN44 & A11-1 & 113.35 & 73.52 & 627 & Chionodraco myersi & $\begin{array}{l}\text { Chionodraco } \\
\text { myersi }\end{array}$ & DQ526430.1 & 99.70 & OK493710 \\
\hline AN45 & A11-1 & 113.35 & 73.52 & 627 & Chionodraco myersi & $\begin{array}{l}\text { Chionodraco } \\
\text { myersi }\end{array}$ & DQ526430.1 & 99.56 & OK493711 \\
\hline AN46 & A11-1 & 113.35 & 73.52 & 627 & Macrourus whitsoni & $\begin{array}{l}\text { Macrourus } \\
\text { whitsoni }\end{array}$ & MT157320.1 & 97.99 & OK493712 \\
\hline AN47 & A11-1 & 113.35 & 73.52 & 627 & Macrourus whitsoni & $\begin{array}{l}\text { Macrourus } \\
\text { whitsoni }\end{array}$ & MT157320.1 & 100.00 & OK493713 \\
\hline AN48 & A4-3 & 112.99 & 72.91 & 438 & Chaenodraco wilsoni & $\begin{array}{l}\text { Chaenodraco } \\
\text { wilsoni }\end{array}$ & NC_039158.1 & 99.24 & OK493714 \\
\hline AN49 & A11-1 & 113.35 & 73.52 & 627 & $\begin{array}{l}\text { Dolloidraco } \\
\text { longedorsalis }\end{array}$ & $\begin{array}{l}\text { Dolloidraco } \\
\text { longedorsalis }\end{array}$ & NC_057667.1 & 99.56 & OK493715 \\
\hline AN50 & A11-1 & 113.35 & 73.52 & 627 & $\begin{array}{l}\text { Dolloidraco } \\
\text { longedorsalis }\end{array}$ & $\begin{array}{l}\text { Dolloidraco } \\
\text { longedorsalis }\end{array}$ & NC_057667.1 & 99.56 & OK493716 \\
\hline AN51 & A11-1 & 113.35 & 73.52 & 627 & $\begin{array}{l}\text { Dolloidraco } \\
\text { longedorsalis }\end{array}$ & $\begin{array}{l}\text { Dolloidraco } \\
\text { longedorsalis }\end{array}$ & NC_057667.1 & 99.56 & OK493717 \\
\hline
\end{tabular}

Note: Morphological names in bold are misidentified samples using morphological taxonomy. 


\begin{tabular}{|c|c|c|c|c|c|c|c|c|c|}
\hline $\begin{array}{l}\text { Sample } \\
\text { No. }\end{array}$ & $\begin{array}{l}\text { Sample } \\
\text { site }\end{array}$ & $\begin{array}{l}\text { Longitude } \\
\left({ }^{\circ} / \mathrm{W}\right)\end{array}$ & $\begin{array}{l}\text { Latitude } \\
\left({ }^{\circ} / \mathrm{S}\right)\end{array}$ & $\begin{array}{l}\text { Sample } \\
\text { Depth(m) }\end{array}$ & Molecular identification & $\begin{array}{l}\text { Morphological } \\
\text { identification }\end{array}$ & $\begin{array}{l}\text { Genbank } \\
\text { voucher No. }\end{array}$ & $\begin{array}{l}\text { Similarity } \\
\text { (\%) }\end{array}$ & $\begin{array}{l}\text { Genbank } \\
\text { No. }\end{array}$ \\
\hline AN52 & A11-2 & 115.10 & 73.02 & 693 & $\begin{array}{l}\text { Dolloidraco } \\
\text { longedorsalis }\end{array}$ & $\begin{array}{l}\text { Dolloidraco } \\
\text { longedorsalis }\end{array}$ & NC_057667.1 & 99.71 & OK493718 \\
\hline AN53 & A4-3 & 112.99 & 72.91 & 438 & Artedidraco lonnbergi & $\begin{array}{l}\text { Artedidraco } \\
\text { lonnbergi }\end{array}$ & HQ712823.1 & 100.00 & OK493719 \\
\hline AN54 & A11-1 & 113.35 & 73.52 & 627 & $\begin{array}{l}\text { Trematomus cf. } \\
\text { lepidorhinus/loennbergi }\end{array}$ & $\begin{array}{l}\text { Trematomus } \\
\text { loennbergii }\end{array}$ & NC_048965.1 & 99.27 & OK493720 \\
\hline AN55 & A4-3 & 112.99 & 72.91 & 438 & $\begin{array}{l}\text { Trematomus cf. } \\
\text { lepidorhinus/loennbergi }\end{array}$ & $\begin{array}{l}\text { Trematomus } \\
\text { loennbergii }\end{array}$ & NC_048965.1 & 99.56 & OK493721 \\
\hline AN56 & A11-1 & 113.35 & 73.52 & 627 & Akarotaxis nudiceps & $\begin{array}{l}\text { Akarotaxis } \\
\text { nudiceps }\end{array}$ & NC_057664.1 & 99.09 & OK493722 \\
\hline AN57 & A11-1 & 113.35 & 73.52 & 627 & Akarotaxis nudiceps & $\begin{array}{l}\text { Akarotaxis } \\
\text { nudiceps }\end{array}$ & NC_057664.1 & 99.41 & OK493723 \\
\hline AN58 & A4-3 & 112.99 & 72.91 & 438 & $\begin{array}{l}\text { Ophthalmolycus } \\
\text { amberensis }\end{array}$ & $\begin{array}{l}\text { Ophthalmolycus } \\
\text { amberensis }\end{array}$ & JN641043.1 & 100.00 & OK493724 \\
\hline AN59 & A11-4 & 117.32 & 72.25 & 523 & Gerlachea australis & $\begin{array}{l}\text { Gerlachea } \\
\text { australis }\end{array}$ & NC_057668.1 & 99.56 & OK493725 \\
\hline AN60 & A4-3 & 112.99 & 72.91 & 438 & Macrourus whitsoni & $\begin{array}{l}\text { Macrourus } \\
\text { whitsoni }\end{array}$ & MT157320.1 & 99.56 & OK493726 \\
\hline AN61 & A11-1 & 113.35 & 73.52 & 627 & Dacodraco hunteri & $\begin{array}{l}\text { Dacodraco } \\
\text { hunteri }\end{array}$ & HQ712963.1 & 99.85 & OK493727 \\
\hline AN62 & A4-3 & 112.99 & 72.91 & 438 & Chaenodraco wilsoni & $\begin{array}{l}\text { Chaenodraco } \\
\text { wilsoni }\end{array}$ & NC_039158.1 & 98.69 & OK493728 \\
\hline AN63 & A4-3 & 112.99 & 72.91 & 438 & $\begin{array}{l}\text { Trematomus cf. } \\
\text { lepidorhinus/loennbergi }\end{array}$ & $\begin{array}{l}\text { Trematomus } \\
\text { loennbergii }\end{array}$ & NC_048965.1 & 99.41 & OK493730 \\
\hline AN64 & A4-3 & 112.99 & 72.91 & 438 & $\begin{array}{l}\text { Trematomus cf. } \\
\text { lepidorhinus/loennbergi }\end{array}$ & $\begin{array}{l}\text { Trematomus } \\
\text { loennbergii }\end{array}$ & HQ713304.1 & 99.85 & OK493731 \\
\hline AN65 & A11-1 & 113.35 & 73.52 & 627 & Chionodraco myersi & $\begin{array}{l}\text { Chionodraco } \\
\text { myersi }\end{array}$ & DQ526430.1 & 99.56 & OK493732 \\
\hline AN66 & A11-1 & 113.35 & 73.52 & 627 & $\begin{array}{l}\text { Vomeridens } \\
\text { infuscipinnis }\end{array}$ & $\begin{array}{l}\text { Vomeridens } \\
\text { infuscipinnis }\end{array}$ & HQ713358.1 & 100.00 & OK493740 \\
\hline AN67 & A11-1 & 113.35 & 73.52 & 627 & Macrourus whitsoni & $\begin{array}{l}\text { Macrourus } \\
\text { whitsoni }\end{array}$ & MT157320.1 & 99.70 & OK493741 \\
\hline AN68 & A11-1 & 113.35 & 73.52 & 627 & Akarotaxis nudiceps & $\begin{array}{l}\text { Akarotaxis } \\
\text { nudiceps }\end{array}$ & NC_057664.1 & 99.70 & OK493743 \\
\hline AN69 & A4-3 & 112.99 & 72.91 & 438 & Gerlachea australis & $\begin{array}{l}\text { Gerlachea } \\
\text { australis }\end{array}$ & NC_057668.1 & 99.70 & OK493745 \\
\hline
\end{tabular}

We used two DNA identification methods to access taxonomic units: assemble species by automatic partitioning (ASAP) (Puillandre et al. 2021)and Bayesian phylogenetics and phylogeography (BPP)(Yang et al. 2014) to infer putative species boundaries based on the COI gene. ASAP uses single locus sequence alignments to create species partitions; it is based on the implementation of a hierarchical clustering algorithm and compares only pairwise genetic distances. All aligned COI sequences were calculated by the ASAP (https://bioinfo.mnhn.fr/abi/public/asap/asapweb.html), with JC69 (Jukes-Cantor) model to compute the distance and default settings (split groups below probability 0.01 , keep 10 best scores). BPP is a Bayesian Markov chain Monte Carlo (MCMC) tool for analysing DNA sequences under the multispecies coalescent (MSC) model. The ultra-metric tree with haplotypes was reconstructed using BEAST v1.10.4 (Drummond et al. 2012). The parameters in BEAUti use the GTR model and gamma shape site model. The number of gamma categories is 4 , uncorrelated relaxed clock, and a chain length of 30000000 iterations for MCMC.

Genetic distances were calculated using the Kimura two-parameter (K2P) distance model (Kimura 1980) with 1000 bootstrap replicates and uniform rates using MEGA X (Kumar et al. 2018). Including intra- and interspecies genetic distances and pairwise distance. We used the online tool SMS to find suitable models of nucleotide substitution under the Akaike information criterion (AIC). BI tree and $\mathrm{ML}$ tree were used to construct the phylogenetic relationships. BI tree was constructed using MrBayes v3.1.2 (Huelsenbeck et al. 2001), and MCMC analysis was run with 10000000 generations, sampling every 1000 generations. We used PhyML3.0 (Guindon et al. 2010) to build an ML tree with GTR and 0.186 gamma shape parameters as substitution models, NII for tree improvement, aLRT SH-like fast likelihood method. Finally, the majority-rule consensus tree was reconstructed and displayed using Figtree v1.4.4. 


\section{Results}

\subsection{Morphological and DNA identification}

A total of 69 fish samples were collected in this study. Most of them were adults and well preserved, but some individuals were small or damaged during preservation and thus difficult to identify. We followed Gon's classification method (Graeme et al. 1992). Limited by the poor Antarctic fish classification literature, the probability of misidentification is greatly increased. In this study, 12 fish species were identified by morphological characters and keys, but 1 species was identified incorrectly as a sister species (Table 1).

All COI fragments were successfully amplified and sequenced. The sequences of the COI gene with high quality (no double peaks, short fragments or background noise) were aligned and contained no insertions, deletions,or stop codons. The length of the COI sequences was $652 \mathrm{bp}$ after alignment, including 237 polymorphic sites (223 parsimony-informative sites, 14 singleton variable sites). The average base composition was $\mathrm{A}=21.03 \%, \mathrm{C}=$ $27.90 \%, G=19.71 \%$, and $\mathrm{T}=31.36 \%$ on average, with a slight bias against $\mathrm{G}$ and $\mathrm{C}$. The best classification result in ASAP (second-best model) supported 69 sequences that represent 11 taxonomic units. Artedidraco lonnbergi and Dolloidraco longedorsalis suggested being one taxonomic unit. Lycenchelys sp. and Ophthalmolycus amberensis were also in the same situation. However, BPP shows a different result with ASAP (Fig. 2). BPP confirmed that $69 \mathrm{COI}$ sequences belonged to 13 taxonomic units, and this result is basically consistent with the result of traditional morphological identification. Altogether, molecular methods proved that 69 sequences belonged to 13 species of fish, 12 genera, 6 families, and 2 orders (Table 2).

Table 2

Fish fauna of the Amundsen Sea in 36th CHINARE

\begin{tabular}{|lllll|}
\hline Order & Family & Species & Amundsen Sea & Record \\
Amundsen Sea
\end{tabular}

\subsection{Genetic distance and phylogeny analysis}

The uncorrected K2P pairwise distance within species was below $1 \%$, averaged $0.31 \%$, and ranged from 0 1.01\%. The genetic distance between species varied between $1.84 \%$ 29.9\% (Fig. 3). The best-fitting model was GTR +G and gamma distribution shape parameter 0.186 . Two phylogenetic trees, BI tree and ML tree, showed a similar topology, and the majority-rule consensus tree was used to show the phylogenetic relationship of fish. The tree supported a branch of Bathydracinidae nested within Channichthyidae. Most individuals in the tree clustered together in groups of the same species.

\section{Discussion}

\subsection{Effectiveness of COI barcoding and species delimitation}

The accuracy of DNA barcoding is the key to species identification, which depends on the degree of intra- and interspecific variation of the selected gene fragments. The less intra- and interspecific overlap there is, the more effective the barcoding. Intraspecific variations are generally similar among species (Waugh 2007). However, the range of interspecific differences varies depending on the size of the selected group and geographic populations. The use of means for intraspecific and interspecific genetic distance comparisons does not allow the detection of problematic cases. Therefore, we compared the minimum interspecific distance with the maximum intraspecific genetic distance (Meier et al. 2008). In this study, the minimum interspecific distance was $1.84 \%$, the maximum intraspecific genetic distance was $1.01 \%$, and the barcoding gap was between $1.01 \%$ and $1.84 \%$. 
With the two different methods we used to infer the putative species boundaries, namely ASAP and BPP. ASAP is based on single-marker pairwise genetic distance and avoids the heavy computational burden of phylogenetic reconstruction. It does not require any biological priori insights and can quickly come up with relevant species hypotheses (Puillandre et al. 2021). BPP can accurately assign species identity at the species level without knowing species boundaries in advance, even when analysis rare taxa with only one locus available(Yang \& Rannala 2017). The classification of most species is consistent. BPP and morphology have obtained similar results, while ASAP has some differences. As the BPP results were consistent with the BLAST results against the Genbank database, BPP was likely to show more accurate species identification results. However, it is worth noting that there are ten results displayed by ASAP. We only consider the classification results of the first- and second-best scores. If barcoding gaps or other prior conditions are considered, ASAP can achieve the same results as BPP. Overall, DNA identification can provide simple and reliable species classification results and shows the uniqueness of the method when morphology is difficult to perform.

\subsection{Phylogenetic relationships}

The COI gene is a short nucleotide fragment from mitochondria and is not the best choice for phylogenetic analysis; however, the topology of its phylogenetic tree might still have reference value (Steinke et al. 2009). The tree topology based on COI barcoding is usually related to the delineation of clusters. Although the ML tree was based on a priori inference and Bayesian inference was based on a posteriori inference, the topology supported by the results was basically the same (Fig. 4). In particular, they both supported that Bathydracinidae were paraphyletic. Previous studies also reported similar results (Derome et al. 2002; Bargelloni et al. 2004). Multiple nuclear markers and multiple studies also confirmed that Bathydracinidae were paraphyletic (Near et al. 2004; Rock et al. 2008). In terms of the phylogenetic relationship, our COI-based phylogenetic signal further verifies the topological structure revealed by other studies.

\subsection{The demersal fish fauna in the Amundsen Sea}

In recent decades, with the deepening of research and the emergence of commercial fishing, increasing information about the community structure and classification of fish in the Southern Ocean has been discovered. In general, Notothenioidei, including Artedidraconidae, Bathydraconidae, Channichthyidae, Harpagiferidae, and Nototheniidae, has an absolute advantage in terms of number, accounting for most of the total species biodiversity (Eastman \& McCune 2000; Eastman 2004). Additionally, there are some typical deep-sea fish groups, such as Liparidae and Zoarcidae. Some Antarctic fish diversity studies based on molecular taxonomy have been applied in the Ross Sea (Smith et al. 2012), Prydz Bay (Li et al. 2018), Scotia Sea (Rock et al. 2008), The Dumont d'Urville Sea (Dettai et al. 2011), and Antarctic Peninsula (Mabragaña et al. 2016) and verified the aforementioned Antarctic fish diversity pattern.

In this study, 13 species of fish were identified in the surveyed seas, most of which belonged to Artedidraconidae, Bathydraconidae, Channichthyidae, and Nototheniidae in addition to Liparidae and Zoarcidae. Harpagiferiade did not appear in our study because these species are usually distributed in the sub-Antarctic region (Navarro et al. 2019), but the Amundsen Sea is located at high latitudes. Relatively speaking, there were only a few sampling stations with shallow sampling depths, which may be the reason why we missed those typical deep-sea groups. At present, the fish fauna of the Amundsen Sea area have been studied by underwater observations. Our results supported that Notothenioidei dominates both in abundance and biomass. This is consistent with the aforementioned general pattern of the Southern Ocean fish fauna. The fish we caught were also roughly similar to the fauna observed by Eastman et al(2012), however, our study provided more detailed species assignment at the species level, with some additional exclusive species recorded. In particular, Ophthalmolycus amberensis, Chaenodraco wilsoni, Dacodraco hunteri, Akarotaxis nudiceps, Artedidraco lonnbergi and Vomeridens infuscipinnis might be recorded for the first time in the Amundsen Sea. It should also be noted that Eastman's data came from underwater photography, and some species are difficult to identify by morphology, in contrast, our results are based on molecular taxonomy analysis of fish catches. From this perspective, our identification results are undoubtedly more credible.

To the best of our knowledge, our study is the first on the molecular taxonomy of fish in the Amundsen Sea. Our results provide important taxonomic information on the demersal fish fauna in the Amundsen Sea. This is of great significance for understanding the biodiversity, taxonomy and biogeography of fish in the Amundsen Sea. However, we believe there are still many unknowns about the diversity of demersal fish in this area that need to be explored. Broader sampling of latitudes, deeper sampling depths, and higher sampling densities are all necessary for future research. Finally, the integration of molecular identification and morphological identification is suggested to ensure precise taxonomy in future studies of Antarctic fishes.

\section{Conclusions}

Based on fish samples collected during the 36th CHINARE, DNA barcoding analysis revealed 13 species belonging to six families (mainly composed of Notothenioidei). We used two different DNA identification methods (ASAP and BPP), combined with barcode gap analysis to determine the species boundary. At the same time, phylogenetic trees of two different algorithms were reconstructed to determine the phylogenetic relationship of Antarctic fish. The evolutionary relationships of Antarctic fish shown in the $\mathrm{ML}$ tree and $\mathrm{BI}$ tree based on $\mathrm{COI}$ in this paper are also consistent with the results of previous phylogenetic studies to some extent. In general, our research provides a more comprehensive description of the molecular taxonomy and diversity of demersal fish in the Amundsen Sea and confirms that DNA barcoding is an effective supplementary method for the identification of most species and can be applied to biodiversity surveys of Antarctic fish. Our study is also the first report to identify the fish fauna in the Amundsen Sea using molecular methods. To fully understand the fish diversity in the Amundsen Sea, more specimens from the broader sampling of latitudes, deeper sampling depths, and higher sampling densities should be collected. In future studies, molecular identifications are suggested to be integrated to and morphological identification of Antarctic fishes to obtain more accurate identification results. 


\section{Declarations}

\section{Acknowledgment}

This work was supported by the "Impact and Response of Antarctic Seas to Climate Change" (IRASCC2020-2022-N0.01-02-02B \& 02-03), Ministry of Natural Resources of the People's Republic of China, Chinese Arctic and Antarctic Administration. We thank all the teammates and crew of the XUELONG R/V for their efforts in collecting specimens during the CHINARE-36 cruise.

\section{Credit author statement}

Shuai Cao: Conceptualization, Data curation, Writing-original draft preparation. Yuan Li: Data curation, Software, Writing- reviewing and editing. Ran

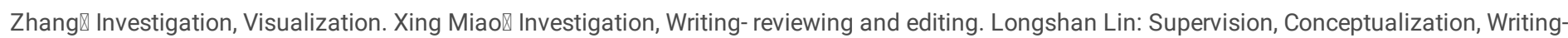
reviewing and editing, Resources. Hai Li: Supervision, Conceptualization, Writing-original draft preparation, Methodology, Data curation, Validation.

\section{References}

1. Allison LC, Johnson HL, Marshall DP, Munday DR (2010) Where do winds drive the Antarctic Circumpolar Current?Geophysical Research Letters,37

2. Alt KG, Cunze S, Kochmann J, Klimpel SJAP (2021) Parasites of Three Closely Related Antarctic Fish Species (Teleostei: Nototheniinae) from Elephant Island. 1-15

3. Bargelloni L, Zane L, Derome N, Lecointre G, Patarnello T (2004) Molecular zoogeography of Antarctic euphausiids and notothenioids: from species phylogenies to intraspecific patterns of genetic variation. Antarct Sci 12:259-268

4. Barnes DK, Souster TJNCC (2011) Reduced survival of Antarctic benthos linked to climate-induced iceberg scouring. 1:365-368

5. Clarke A, Johnston IA (1996) Evolution and adaptive radiation of Antarctic fishes. Trends Ecol Evol 11:212-218

6. De Broyer C, Koubbi P, Griffiths H, Grant SA (2014) Biogeographic atlas of the Southern Ocean. Scientific Committee on Antarctic Research Cambridge

7. Derome N, Chen W-J, Dettaı A, Bonillo C, Lecointre G (2002) Phylogeny of Antarctic dragonfishes (Bathydraconidae, Notothenioidei, Teleostei) and related families based on their anatomy and two mitochondrial genes. Mol Phylogenet Evol 24:139-152

8. Dettai A, Adamowizc SJ, Allcock L, Arango CP, Barnes DKA, Barratt I, Chenuil A, Couloux A, Cruaud C, David B, Denis F, Denys G, Díaz A, Eléaume M, Féral J-P, Froger A, Gallut C, Grant R, Griffiths HJ, Held C, Hemery LG, Hosie G, Kuklinski P, Lecointre G, Linse K, Lozouet P, Mah C, Monniot F, Norman MD, O'Hara T, Ozouf-Costaz C, Piedallu C, Pierrat B, Poulin E, Puillandre N, Riddle M, Samadi S, Saucède T, Schubart C, Smith PJ, Stevens DW, Steinke D, Strugnell JM, Tarnowska K, Wadley V, Ameziane N (2011) DNA barcoding and molecular systematics of the benthic and demersal organisms of the CEAMARC survey. Polar Sci 5:298-312

9. Drummond AJ, Suchard MA, Xie D, Rambaut A (2012) Bayesian Phylogenetics with BEAUti and the BEAST 1.7. Molecular Biology and Evolution, 29, 1969-1973

10. Eastman J, McCune AJJoFB (2000) Fishes on the Antarctic continental shelf: evolution of amarine species flock? 57:84-102

11. Eastman JT (2000) Antarctic notothenioid fishes as subjects for research in evolutionary biology. Antarct Sci 12:276-287

12. Eastman JT (2004) The nature of the diversity of Antarctic fishes. Polar Biol 28:93-107

13. Eastman JT, Amsler MO, Aronson RB, Thatje S, McClintock JB, Vos SC, Kaeli JW, Singh H, La Mesa M (2012) Photographic survey of benthos provides insights into the Antarctic fish fauna from the Marguerite Bay slope and the Amundsen Sea. Antarct Sci 25:31-43

14. Francis CM, Borisenko AV, Ivanova NV, Eger JL, Lim BK, Guillen-Servent A, Kruskop SV, Mackie I, Hebert PD (2010) The role of DNA barcodes in understanding and conservation of mammal diversity in southeast Asia.PLoS One, 5, e12575

15. Graeme B, PJRiF F (1992) Fishes of the Southern Ocean

16. Griffiths HJ, Danis B, Clarke A (2011) Quantifying Antarctic marine biodiversity: The SCAR-MarBIN data portal. Deep Sea Research Part II: Topical Studies in Oceanography, 58, 18-29

17. Guindon S, Dufayard J-F, Lefort V, Anisimova M, Hordijk W, Gascuel OJSb (2010) New algorithms and methods to estimate maximum-likelihood phylogenies: assessing the performance of PhyML 3.0. 59:307-321

18. Gutt J, Piepenburg DJMEPS (2003) Scale-dependent impact on diversity of Antarctic benthos caused by grounding of icebergs. 253:77-83

19. Haumann FA, Gruber N, Münnich M, Frenger I, Kern SJN (2016) Sea-ice transport driving Southern Ocean salinity and its recent trends. 537:89-92

20. Hebert PD, Cywinska A, Ball SL, deWaard JR (2003a) Biological identifications through DNA barcodes. Proc Biol Sci 270:313-321

21. Hebert PD, Ratnasingham S, deWaard JR (2003b) Barcoding animal life: cytochrome c oxidase subunit 1 divergences among closely related species. Proc Biol Sci 270(Suppl 1):S96-99

22. Hebert PD, Stoeckle MY, Zemlak TS, Francis CM (2004) Identification of Birds through DNA Barcodes. PLoS Biol 2:e312

23. Hellberg RS, Kawalek MD, Van KT, Shen Y, Williams-Hill DM (2014) Comparison of DNA Extraction and PCR Setup Methods for Use in HighThroughput DNA Barcoding of Fish Species. Food Anal Methods 7:1950-1959 
24. Hubert N, Meyer CP, Bruggemann HJ, Guerin F, Komeno RJ, Espiau B, Causse R, Williams JT, Planes S (2012) Cryptic diversity in Indo-Pacific coralreef fishes revealed by DNA-barcoding provides new support to the centre-of-overlap hypothesis.PLoS One, 7, e28987

25. Huelsenbeck JP, Ronquist F, Nielsen R, Bollback, JPJs (2001) Bayesian inference of phylogeny and its impact on evolutionary biology. 294, 23102314

26. Hunt GL Jr, Stabeno P, Walters G, Sinclair E, Brodeur RD, Napp JM, Bond NAJDSRPITSiO (2002) Climate change and control of the southeastern Bering Sea pelagic ecosystem. 49:5821-5853

27. Joyner CCJUoSCP, Columbia SC (1998) 369 Governing the frozen commons: The Antarctic regime and environmental protection. 1998

28. Jun S-Y, Kim J-H, Choi J, Kim S-J, Kim B-M, An S-I (2020) The internal origin of the west-east asymmetry of Antarctic climate change. Sci Adv 6:eaaz1490

29. Keller DP, Kriest I, Koeve W, Oschlies AJGRL (2016) Southern Ocean biological impacts on global ocean oxygen. 43:6469-6477

30. Kim S-Y, Lim D, Rebolledo L, Park T, Esper O, Muñoz P, La HS, Kim TW, Lee S (2021) A 350-year multiproxy record of climate-driven environmental shifts in the Amundsen Sea Polynya, Antarctica. Global and Planetary Change, p 205

31. Kimura M (1980) A simple method for estimating evolutionary rates of base substitutions through comparative studies of nucleotide sequences. J Mol Evol 16:111-120

32. Kumar S, Stecher G, Li M, Knyaz C, Tamura K (2018) MEGA X: molecular evolutionary genetics analysis across computing platforms. Mol Biol Evol 35:1547

33. La HS, Park K, Wahlin A, Arrigo KR, Kim DS, Yang EJ, Atkinson A, Fielding S, Im J, Kim TW, Shin HC, Lee S, Ha HK (2019) Zooplankton and micronekton respond to climate fluctuations in the Amundsen Sea polynya. Antarctica Sci Rep 9:10087

34. Lakra WS, Verma MS, Goswami M, Lal KK, Mohindra V, Punia P, Gopalakrishnan A, Singh KV, Ward RD, Hebert P (2011) DNA barcoding Indian marine fishes. Mol Ecol Resour 11:60-71

35. Lara A, Ponce de Leon JL, Rodriguez R, Casane D, Cote G, Bernatchez L, Garcia-Machado E (2010) DNA barcoding of Cuban freshwater fishes: evidence for cryptic species and taxonomic conflicts. Mol Ecol Resour 10:421-430

36. Li Y, Zhang L, Song P, Zhang R, Wang L, Lin L (2018) Fish diversity and molecular taxonomy in the Prydz Bay during the 29th CHINARE. Acta Oceanol Sin 37:15-20

37. Mabragaña E, Delpiani S, Rosso J, González-Castro M, Antoni MD, Hanner R, de Astarloa JDJDBiMP (2016) Barcoding Antarctic fishes: species discrimination and contribution to elucidate ontogenetic changes in Nototheniidae.213-242

38. McGlone MS, Turney CS, Wilmshurst JM, Renwick J, Pahnke KJNG (2010) Divergent trends in land and ocean temperature in the Southern Ocean over the past 18,000 years. $3,622-626$

39. Meier R, Zhang G, Ali F (2008) The use of mean instead of smallest interspecific distances exaggerates the size of the "barcoding gap" and leads to misidentification. Syst Biol 57:809-813

40. Mikkelsen NT, Schander C, Willassen E (2007) Local scale DNA barcoding of bivalves (Mollusca): a case study. Zoolog Scr 36:455-463

41. Mintenbeck K, Barrera-Oro ER, Brey T, Jacob U, Knust R, Mark FC, Moreira E, Strobel A, Arntz WE (2012) Impact of Climate Change on Fishes in Complex Antarctic Ecosystems. Global Change in Multispecies Systems Part 1. p.^pp. 351-426

42. Mintenbeck K, Torres JJ (2017) Impact of Climate Change on the Antarctic Silverfish and Its Consequences for the Antarctic Ecosystem. The Antarctic Silverfish: a Keystone Species in a Changing Ecosystem. p.^pp.253-286

43. Murphy KR, Kalmanek EA, Cheng CHC (2016) Diversity and biogeography of larval and juvenile notothenioid fishes in McMurdo Sound, Antarctica. Polar Biol 40:161-176

44. Navarro JM, Paschke K, Ortiz A, Vargas-Chacoff L, Pardo LM, Valdivia N (2019) The Antarctic fish Harpagifer antarcticus under current temperatures and salinities and future scenarios of climate change. Prog Oceanogr 174:37-43

45. Near TJ, Pesavento JJ, Cheng CH (2004) Phylogenetic investigations of Antarctic notothenioid fishes (Perciformes: Notothenioidei) using complete gene sequences of the mitochondrial encoded 16S rRNA. Mol Phylogenet Evol 32:881-891

46. Pineda-Metz SE, Gerdes D, Richter CJNc (2020) Benthic fauna declined on a whitening Antarctic continental shelf. 11:1-7

47. Puillandre N, Brouillet S, Achaz G (2021) ASAP: assemble species by automatic partitioning. Mol Ecol Resour 21:609-620

48. Randall-Goodwin E, Meredith M, Jenkins A, Yager P, Sherrell R, Abrahamsen EP, Guerrero R, Yuan X, Mortlock R, Gavahan KJESotA (2015) Freshwater distributions and water mass structure in the Amundsen Sea Polynya region, AntarcticaFreshwater distributions and water mass structure in the ASP region. 3

49. Rock J, Costa FO, Walker DI, North AW, Hutchinson WF, Carvalho GR (2008) DNA barcodes of fish of the Scotia Sea, Antarctica indicate priority groups for taxonomic and systematics focus. Antarct Sci 20:253-262

50. Sahade R, Lagger C, Torre L, Momo F, Monien P, Schloss I, Barnes DK, Servetto N, Tarantelli S, Tatián MJSA (2015) Climate change and glacier retreat drive shifts in an Antarctic benthic ecosystem. 1, e1500050

51. Smith PJ, Steinke D, Dettai A, McMillan P, Welsford D, Stewart A, Ward RD (2012) DNA barcodes and species identifications in Ross Sea and Southern Ocean fishes. Polar Biol 35:1297-1310

52. Steinke D, Zemlak TS, Boutillier JA, Hebert PDN (2009) DNA barcoding of Pacific Canada's fishes. Mar Biol 156:2641-2647

Page 10/14 
53. Tynan CT (1998) Ecological importance of the southern boundary of the Antarctic Circumpolar Current. Nature 392:708-710

54. Vander Zanden MJ, Vadeboncoeur Y, Chandra SJE (2011) Fish reliance on littoral-benthic resources and the distribution of primary production in lakes. 14:894-903

55. Waugh J (2007) DNA barcoding in animal species: progress, potential and pitfalls. BioEssays 29:188-197

56. Yager PL, Sherrell RM, Stammerjohn SE, Alderkamp A-C, Schofield O, Abrahamsen EP, Arrigo KR, Bertilsson S, Garay DL, Guerrero RJO (2012) ASPIRE: the Amundsen Sea Polynya international research expedition. 25, 40-53

57. Yang Z, Rannala BJMB, Evolution (2014) Unguided species delimitation using DNA sequence data from multiple loci. 31:3125-3135

58. Yang Z, Rannala BJME (2017) Bayesian species identification under the multispecies coalescent provides significant improvements to DNA barcoding analyses. 26:3028-3036

59. Zhang J-B, Hanner R (2011) DNA barcoding is a useful tool for the identification of marine fishes from Japan. Biochem Syst Ecol 39:31-42

60. Zhang J, Hanner R (2012) Molecular approach to the identification of fish in the South China Sea. PLoS ONE 7:e30621

\section{Figures}

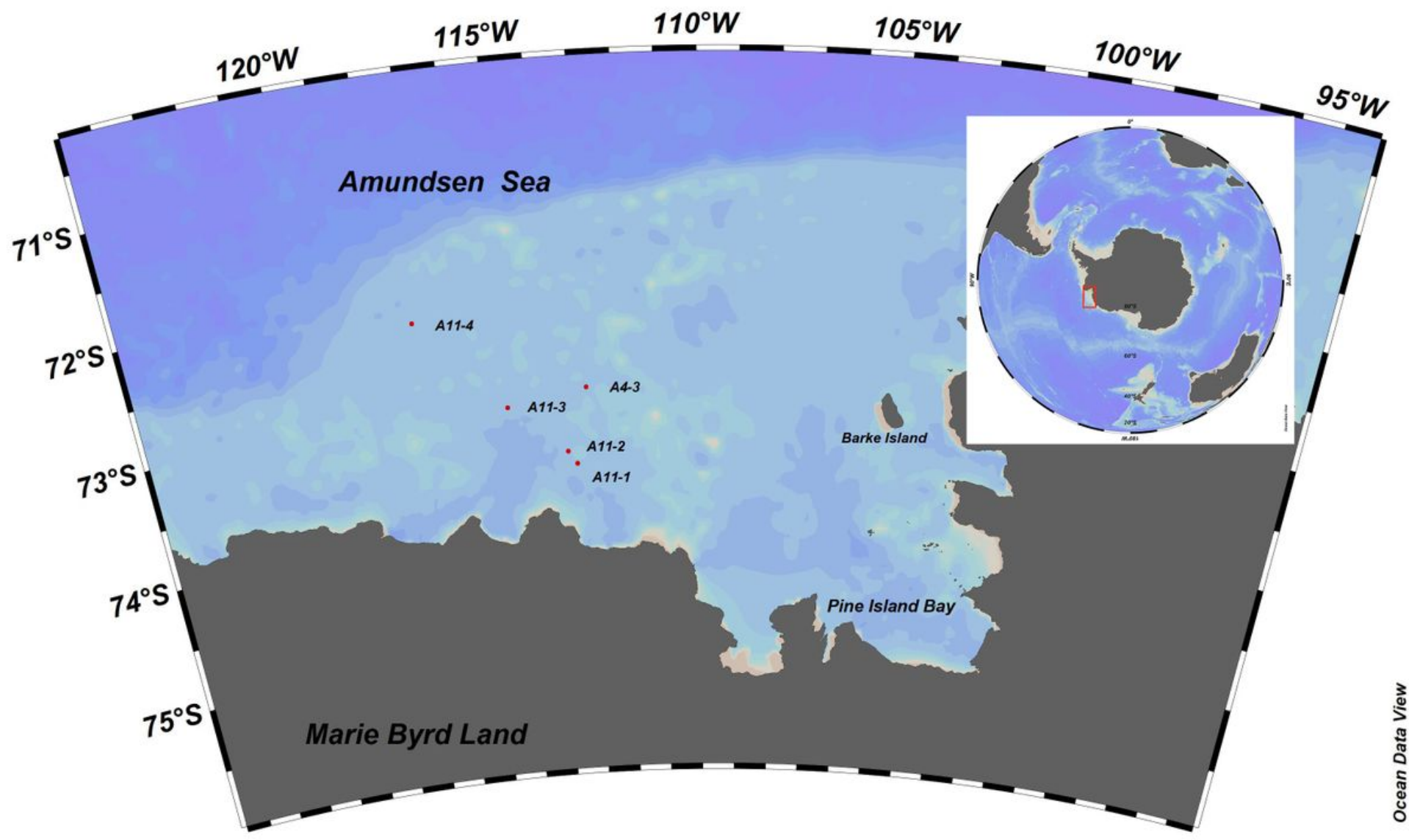

Figure 1

Map of bottom trawl stations of CHINARE-36 cruise in the Amundsen Sea. 


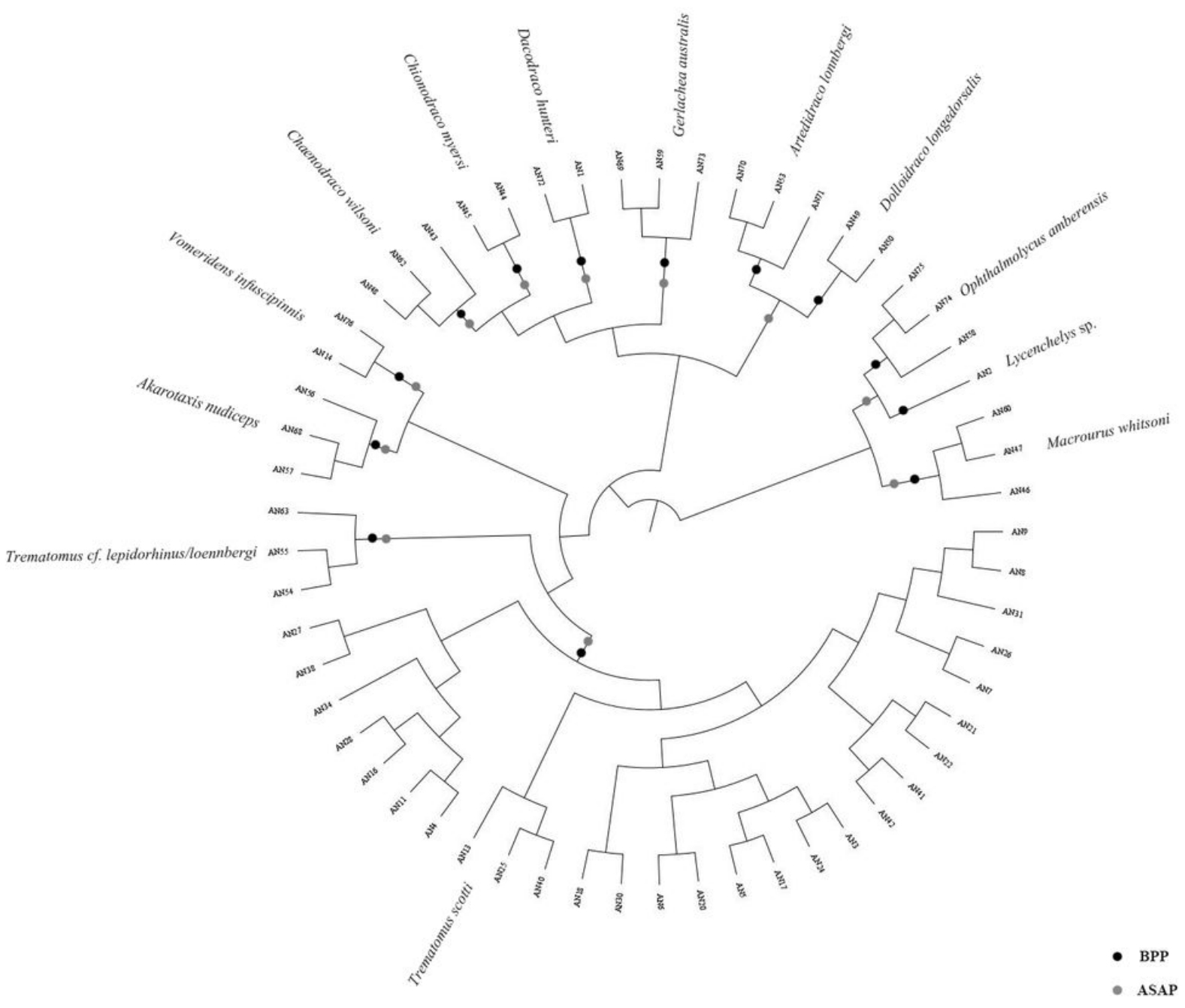

Figure 2

Results of DNA-based classification from ASAP and BPP on COI. The ultra-metric tree with haplotypes was obtained from BEAST. 


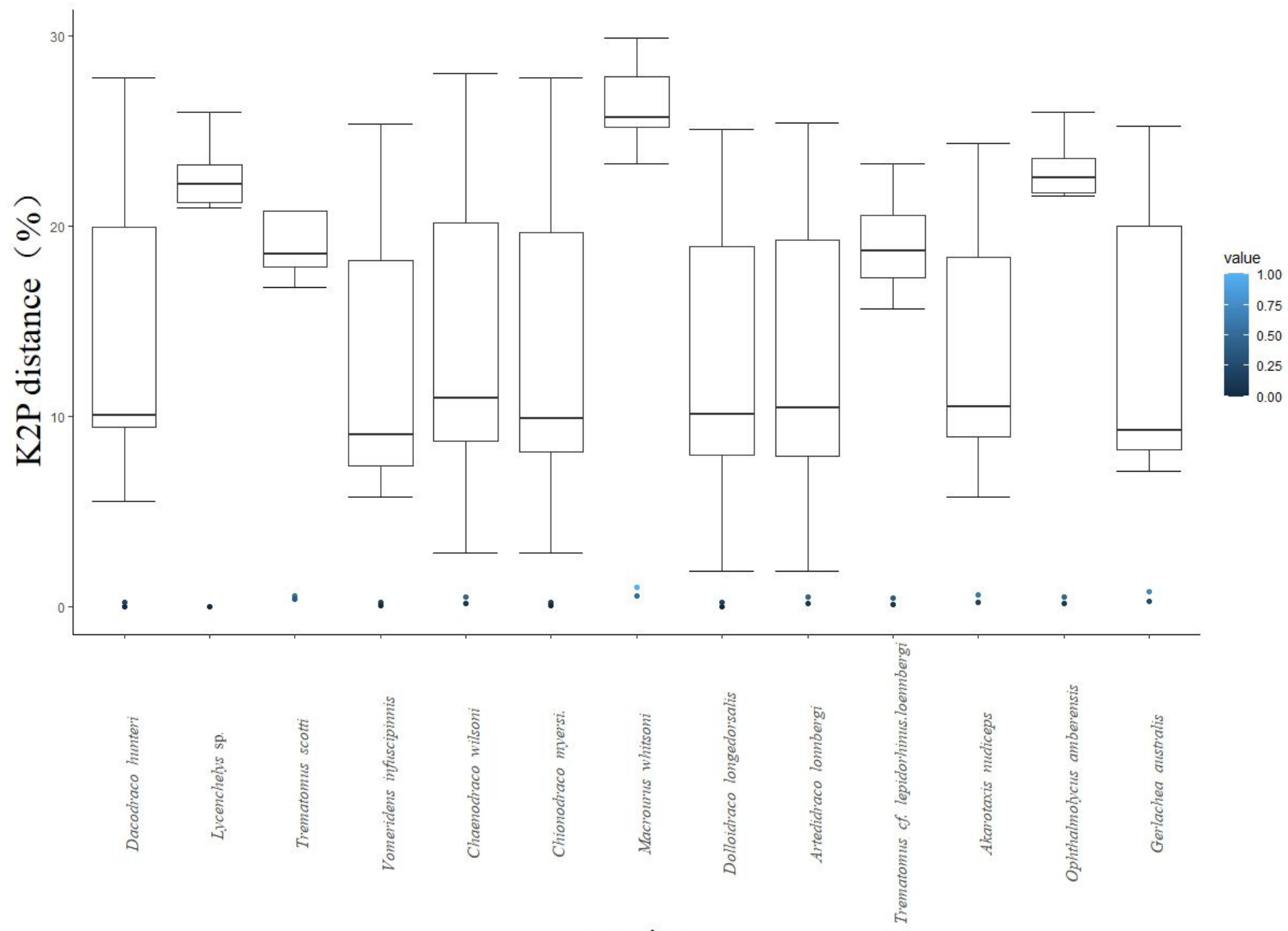

species

Figure 3

DNA barcoding gaps for all species based on the K2P model. Median interspecific distances with maximum and minimum values are represented by the upper and lower bars, respectively. The maximum and the minimum intraspecific genetic distance are represented by blue dots with different color depths. 


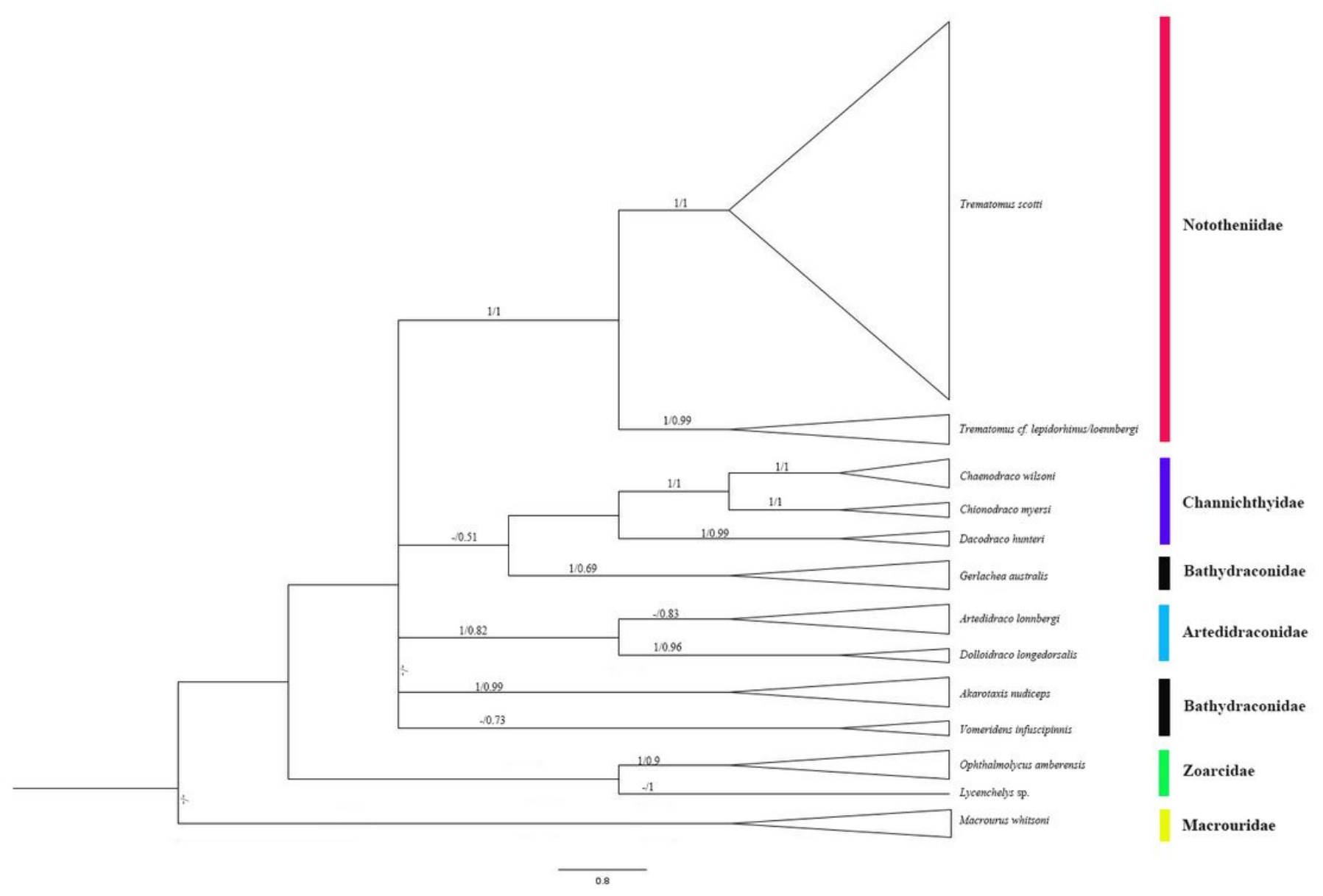

Figure 4

The Bayesian inference COI phylogenetic tree for 69 Antarctic fish in the Amundsen Sea was obtained from MrBayes, with the scale bars proportional to substitution rates; support values are ML Probabilities support/ Bayesian Posterior; ML supports for the clades are also present in the ML trees. 